\title{
Opac-portal: una nueva forma de ofrecer los recursos y servicios de la biblioteca
}

\author{
Por Mª Victoria Játiva Miralles
}

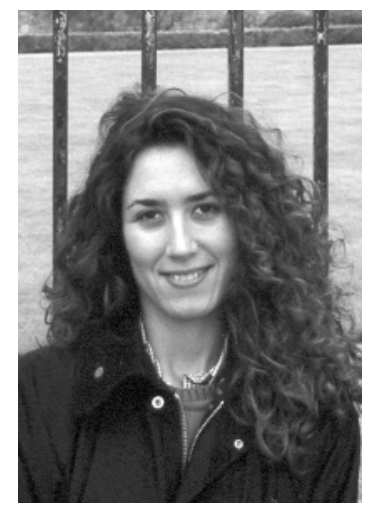

Nov vation bisio Miralles, licenciada en documentación por la Universidad de Murcia. Actualmente, desarrolla su trabajo como responsable de la Biblioteca de Filosofía y LetrasTrabajo Social de la Universidad de Alicante.
Resumen: Se presenta una serie de pautas para el diseño de un opac de última generación que supere y mejores las prestaciones de los opacs actuales y que integre los distintos recursos y servicios de información de la biblioteca.

Palabras clave: Opac extendido, Opac expandido, Opac de última generación, Integración de recursos informativos.

Title: Opac-portal: a new way to provide the library services and resources.

Abstract: Some guidelines are given in order to design a next generation catalogue that improves the actual performances and integrates all the $l i$ brary information resources and services.

Keywords: Extended opac, Expanded opac, Last generation opac, Integration of information resources.

Játiva Miralles, M $\boldsymbol{M}^{a}$ Victoria. «Opac-portal: una nueva forma de ofrecer los recursos y servicios de la biblioteca». En: El profesional de la información, 2002, noviembre-diciembre, v. 11, n. 6, pp. 442-453.

\section{A modo de introducción}

El trabajo que se presenta puede resultar de interés para los profesionales de las bibliotecas y los centros de documentación, así como para los docentes de las escuelas y facultades de ciencias de la documentación. El propósito principal es dar a conocer las pautas para diseñar un opac-portal que presente de manera organizada los recursos de información y los servicios de la biblioteca.

Esta herramienta de consulta puede, y debe, convertirse en el eje informativo de la biblioteca a través de internet, gracias al protocolo de interconexión Ansi Z39.50, las aplicaciones de granularidad (de nivel de detalle) y las posibilidades de DOI (digital object identifier, sistema de codificación por el que los editores científicos pueden identificar los artículos y enlazarlos a otros), ofreciendo un acceso único a todos los recursos y fuentes de información.

Si aúna la búsqueda, la información al usuario y la reproducción de documentos a texto completo, el opac se puede convertir en una plataforma de integración de fuentes de información locales (exclusivas para una comunidad de usuarios concreta) y en línea por medio de una presentación uniforme de la pantalla. Lo importante es conseguir la unión de recursos y fuentes de información eliminando la dispersión y multiplicidad de accesos. Hasta ahora, la consulta y la recuperación de información eran procesos diferentes. Sin embargo, desde la aparición de internet y las posibilidades que ofrece, cambian los procesos y se convierten en acciones consecutivas; buscamos información y la recuperamos en línea de forma completa y no sólo referencias como hasta este momento.

\section{Generaciones de opac}

Según la propuesta hecha por Hildreth, se pueden distinguir tres generaciones de opac:

-1 ${ }^{\text {a }}$ generación. Concebidos según el modelo manual. Permitían el acceso a través de los encabezamientos tradicionales de los catálogos manuales. Carecían de la uniformidad de criterios que más tarde darían los formatos marc, lo cual dificultaba enormemente las búsquedas de los usuarios y el intercambio de registros bibliográficos entre distintas bibliotecas, así como la creación de catálogos colectivos. La finalidad era mejorar el trabajo interno en las bibliotecas.

- $2^{a}$ generación. Son básicamente el resultado de una asociación entre el catálogo tradicional y los sistemas clásicos de recuperación de información bibliográfica en línea. Se caracterizaban por una interfaz más amigable para los usuarios, por la normalización de los métodos de descripción bibliográfica y por la potencia de las herramientas de recuperación. Además de utilizar los ficheros usuales en las bibliotecas (auto- 
res, materias y títulos) permitían la realización de búsquedas por palabras clave en todos los campos, el uso de truncamiento y la limitación de consultas a determinados aspectos o periodos.

$-3^{\mathrm{a}}$ generación. Surgen con el objeto de subsanar las deficiencias observadas en las aplicaciones anteriores. En los años 90, la mayoría de los catálogos en línea van a incorporar la interfaz gráfica de Windows y consiguen mejorar la amigabilidad. A finales de dicha década, con internet, se hace posible la consulta del catálogo independientemente del lugar donde se encuentre el usuario, extendiéndose y popularizándose su uso.

En este período las interfaces son muchos más fáciles de usar. Sus funciones avanzadas permiten optimizar la consulta y modificación de las búsquedas. Además, se mejora la visualización y la navegación a través de los resultados, que se pueden ordenar por distintos métodos. Permiten la integración en el catálogo de otros recursos de información y ofrecen asistencia al usuario e incluso la posibilidad de realizar gestiones a través de él: reservas, dar de alta al usuario, solicitar documentos, etc.

Actualmente las bibliotecas no están formadas solamente por colecciones en soportes tangibles (libros, revistas, cd-rom, vídeos, casetes, etc.) sino que cada día más adquieren, gestionan y ofrecen multitud de documentos en soporte digital de acceso remoto comerciales y gratuitos (bases de datos, revistas-e, libros-e, recursos internet) los cuales no siempre son de su propiedad, sino que sólo poseen la licencia de uso.

\section{Mejorar, ampliar e integrar recursos informativos}

Los opacs pueden ser mejorados con el desarrollo de los recursos informativos que ofrecen, tanto con el

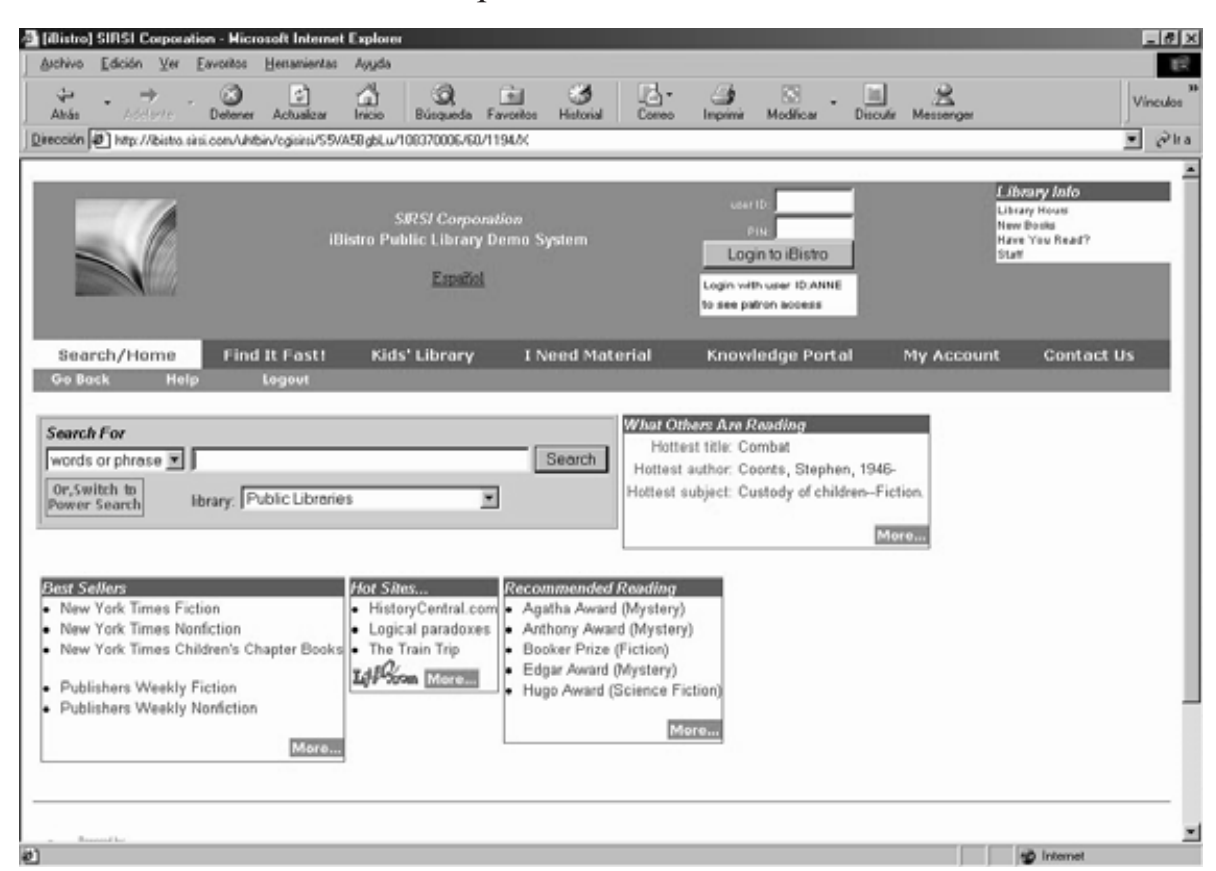

aumento de su contenido como ampliando y extendiendo sus posibilidades de acceso (Peis, FernándezMolina, 1996). Deben crecer en la oferta y variación de los servicios a distancia. El usuario ha de encontrar el nuevo catálogo como un portal de acceso a los distintos recursos y servicios informativos que ofrece la biblioteca, se tiene que convertir en su menú o carta de presentación.

\section{«Con internet se hace posible la consulta del catálogo inde- pendientemente del lugar don- de se encuentre el usuario, ex- tendiéndose y popularizándose su uso»}

Actualmente la consulta de los recursos que el centro suministra resulta complicada debido a la dispersión que hay con múltiples formas de acceso. Se debe tender a integrar diferentes recursos en el opac con un mismo y único procedimiento de utilización, este proceso se llevaría a cabo en 3 niveles (Jiménez, 2001):

-Integración "meta": búsqueda en bases de datos, catálogos, revistas electrónicas, etc.

-Integración "plana": interacción o comunicación del usuario con la biblioteca por medio de servicios de alerta, de reservas, de renovaciones de documentos, etc.

—Integración "profunda": enlaces a los textos completos de distintos materiales.

\section{Opac-portal}

En los últimos tiempos el término portal se ha hecho muy popular, aunque muchas veces se emplea y se le asigna un significado inapropiado. Es simplemente un punto de entrada o acceso común a diversos recursos e informaciones que ofrece la Red. En él, los contenidos temáticos, los servicios, los productos, etc., se agrupan, combinan y organizan de manera que el usuario pueda localizar y utilizar cómodamente todo cuanto necesita sin necesidad de salir de un sitio web. Resulta una forma fácil y rápida para conseguir el acceso a los diversos recursos que con más frecuencia se utilizan. El fuerte crecimiento de los portales se observa ya desde el año 1998. Se puede afirmar que es en estos momentos cuando se 
han convertido en la herramienta básica y principal para organizar y estructurar los contenidos de internet, ayudando y orientando a los usuarios (noveles y/o expertos), los cuales valoran la facilidad de entrada y acceso a la información, así como la organización de contenidos y la navegación.

Existe una clasificación en función del tipo de usuario al que se dirigen, distinguiéndose entre generales (o megaportales), especializados, corporativos y verticales, también conocidos como vortales (García Gómez, Saorín Pérez, 2001).

El opac-portal que se plantea formaría parte del grupo de los denominados corporativos. Se trataría de una sede web cuya misión sería suministrar información y conocimiento personalizado a los miembros de un colectivo de usuarios (en el caso de una biblioteca de universidad, la comunidad universitaria con usuarios reales, virtuales y potenciales). Debe dar acceso a infinidad de recursos tanto internos o propios de la institución como a información en línea externa a ella.

Morrison (2000) ha hecho una curiosa enumeración de las características de un portal. El autor utiliza las iniciales del término y nos ofrece las siguientes pistas para poder identificarlo de entre cualquier otra página web:

—Personalización para usuarios finales;

-Organización del escritorio;

—Recursos informativos divididos y organizados;

— Traza o seguimiento de las actividades de los usuarios;

-Acceso a repositorios de datos;

—Localización de personas o cosas importantes.

Algunas ventajas de los opac-portales serían:

-Diversificación de servicios. Supone un verdadero avance y una plena integración de todo tipo de recursos y fuentes de información con una misma forma de acceso.

- Mejora la navegación y localización de información y conocimiento.

-Concentran toda la información importante respecto a un sector o un ámbito del conocimiento concreto (empleo de accesos de reconocimiento e identificación de usuarios).

—Posibilidad de configuración o personalización de la web atendiendo a las necesidades concretas de cada tipo de usuarios. Lo ideal será que cualquiera lo utilice de manera sistemática, para que se convierta en la única y más útil puerta de entrada.
-Oportunidad de seleccionar contenidos, recursos, servicios, diseños de pantallas, etc., que se ajusten a las necesidades particulares de cada usuario.

La diferencia entre un opac-portal y la sede web de biblioteca estaría en que el primero presenta y reúne de forma organizada los servicios y recursos de/sobre la biblioteca a través de una única vía de entrada. Se trata de una plataforma de integración de las distintas fuentes y los variados servicios de información. Los contenidos se pueden personalizar por tipo de usuario, la información se organiza y es dirigida a través de esta nueva herramienta de presentación de los recursos.

\section{«El usuario debe encontrar el nuevo catálogo como un portal de acceso a los distintos recur- sos y servicios informativos que ofrece la biblioteca»}

Una sede web es una localización digital que se identifica por un url y que contiene una o más páginas asociadas a la portada inicial. No pretende reunir ni estructurar servicios y recursos de forma integradora sino que el usuario debe acceder a distintas webs para localizar la información ya que se encuentra dispersa y no organizada desde una misma plataforma de acceso común a contenidos múltiples.

La tendencia actualmente es personalizar cada vez más los portales y eliminar los distintos menús o formas de acceso para evitar tener que ir navegando de una página a otra hasta localizar la información. Lo deseable es que todo sea accesible desde un misma página web inicial: el opac de la biblioteca, la conexión a bases de datos, el acceso a revistas electrónicas, la información sobre los servicios que ofrece, buscadores de internet, etc. Los usuarios cada vez son más autónomos y deben encontrar la información y los datos que necesitan desde el opac-portal del centro. Por su parte, la biblioteca debe trabajar y ampliar los recursos, servicios y las fuentes personalizadas de información para cada tipo de usuario; por ejemplo, en el caso de una biblioteca universitaria: estudiantes, postgrados, profesores, personal de administración y servicios $\mathrm{y}$ visitantes en general.

En España un ejemplo bastante aproximado de este tipo de opac-portal es el de la biblioteca de la $U C 3 M$, que da la posibilidad de acceder a multitud de recursos integrados y accesibles de forma estructurada desde su página inicial. Desde el propio opac se pueden seleccionar opciones como: "Otros recursos", "Información" o "Servicios al usuario" que dan acceso a múltiples servicios y formas de recuperación de información. Por ejemplo, a partir de "Otros recursos" se 
accede a catálogos Z39.50; a enlaces a páginas web que conectan a su vez a consorcios de bibliotecas, a redes de bibliotecas, etc.; la opción «Recursos electrónicos» permite utilizar bases de datos, revistas electrónicas, sumarios del BOE, etc.; el sub-apartado «Recursos de información en internet» enlaza con fuentes seleccionadas por temas sobre materias como derecho, geografía, turismo, etc. Desde "Información" es posible utilizar la guía de la biblioteca, la oferta de cursos de formación de usuarios, los puntos de servicio, etc. Se trata de una aproximación cercana a la idea que aquí se propone, pero que no se identifica totalmente con el modelo que se plantea.

http://163.117.11.227/uhtbin/cgisirsi/JOY1y6rGPB/21 $183011 / 60 / 69$

Además, la propia web de la biblioteca (que repite muchos contenidos accesibles presentes ya en el opac), presenta ya la idea de "Biblioteca personalizada" desde la selección que hace de servicios y recursos para los distintos colectivos de usuarios de la comunidad universitaria a la que sirve. Aquí se empieza a personalizar e individualizar en la oferta de información.

\section{http://www.uc3m.es/bibliotecal}

No obstante, y en oposición a todo lo expuesto anteriormente, se puede citar el caso del motor de búsqueda Google. Al contrario que la mayor parte de otros servicios semejantes como el índice Yahoo! o el propio Altavista, ha huido de la fórmula de diseño de portal, centrándose en lo que realmente sus usuarios buscan o necesitan: que sea el mejor motor posible sin la oferta de tantos otros servicios añadidos. Su indudable éxito puede hacernos reflexionar acerca de que lo que el usuario final busca es satisfacer unas necesidades de información muy concretas. Sin embargo, esto no se opone totalmente a la propuesta de opac-portal, pues lo que se pretende con él es integrar toda una serie de servicios actualmente dispersos (lo que provoca desorientación y dificultades en la localización de información y conocimiento) y poder encontrarlos con una misma herramienta de búsqueda y localización de información.

La idea que se persigue es convertir el actual catálogo en un «opac extendido", es decir, ampliar de manera considerable las posibilidades que ofrecen los actuales catálogos en línea. Por ejemplo hay librerías electrónicas como Amazon, Barnes \& Noble o Powell's Books que funcionan, en cierto modo, como un opac extendido: tienen enlaces con páginas de críticas literarias, es posible crear perfiles temáticos para recibir información periódicamente, etc. El opac debe convertirse cada día más en un instrumento para que el usuario esté en contacto permanente y directo con la biblioteca. http://www.amazon.co.uk

http://www.barnesandnoble.com/

http://www.powells.com/

\section{Alternativas de presentación de recursos-e}

Hasta ahora las bibliotecas han utilizado básicamente 3 sistemas o formas para mostrar los recursos electrónicos:

\section{Creación de bases de datos}

Una primera opción consiste en la creación de bdds de este tipo de fuentes digitales: de otras bases de datos, revistas-e, libros-e, recursos internet, etc. Son las llamadas subject gateways. Se alimentan y gestionan de forman diferente a como lo hace el catálogo de la biblioteca y la recuperación de información se realiza de acuerdo con las pautas del software propio de cada sistema. Como ejemplo se pueden citar las siguientes:

-En el ámbito español destacan: la bases de datos EP!: webs d'interès politècnic de la biblioteca de la Universitat Politècnica de Catalunya y también la base de datos de recursos de economía y empresa de la Universidad Complutense de Madrid.

http://www.upc.es/catala/biblio/biblio.htm

http://www.ucm.es/BUCM/frames04.htm

- La mayoría de sistemas de bases de datos de recursos web ha sido desarrollada de forma conjunta por bibliotecas universitarias y especializadas. Algunos ejemplos en el entorno de Gran Bretaña son (Estivill, A.; Abadal, E., 2000):

a. Proyecto eLib (Electronic Libraries Program$m e)$. Surgió a partir del informe que Brian Follet en 1993 presentó al Jfc-Lrg (Joint Funding Councils-Libraries Review Group). En el año 1995 se creó el programa eLib gestionado por el Jisc (Joint Information Systems Committee).

http://www.ukoln.ac.uk/services/elib/

b. Resource Discovery Network (RDN). Empezó a funcionar en enero de 1999 y se trata de una red cooperativa que agrupa bases de datos de carácter temático, siendo su finalidad facilitar una consulta simultánea. Hoy en día funciona como portal de acceso a diferentes recursos web de distintas temáticas.

http://www.rdn.ac.uk

c. El proyecto IMesh (actualmente International Collaboration on Internet Subject Gateways) también trabaja en esta misma línea.

http://imesh.org 
d. Por otra parte, Renardus propone crear un modelo de recuperación de información que permita realizar búsquedas conjuntas con una misma interfaz.

http://www.renardus.org/

\section{Todo en el opac}

La segunda opción es la que presenta los recursos digitales integrados en el propio opac por medio de una descripción en formato marc o usando los elementos del Dublin Core. No existe ningún ejemplo de esta opción en las bibliotecas españolas, aunque sí se pueden señalar algunas experiencias elaboradas en esta línea en centros estadounidenses:

-Research Library de Los Alamos National Laboratory.

http://lib-www.lanl.gov/

-Biblioteca de la University of Stanford.

http://www-sul.stanford.edu/search/socii/

-Biblioteca de la University of California, San Diego.

http://roger.ucsd.edu/

—Biblioteca de la Iowa State University.

http://www.lib.iastate.edu/webpac-bin/

wgbroker?new+-access+elib

-Biblioteca de la Ohio University.

http://alice.library.ohiou.edu/

\section{Opac y además guías temáticas}

La tercera alternativa es aquella en la que se presenta simultáneamente una descripción de los recursos digitales en el opac, pero que cuenta además con el diseño de páginas web en forma de guías de recursos web seleccionados por temas o categorías (se componen de listados ordenados alfabéticamente por título o bien organizados por materia). Un ejemplo ya mencionado es la Universitat Politècnica de Catalunya.

Hoy en día en muchas webs de bibliotecas se puede observar cómo todas estas opciones señaladas conviven de forma separada e independiente del opac. Por lo tanto no son excluyentes, sino que se complementan y son distintas alternativas seguidas por las bibliotecas.

\section{Ventajas e inconvenientes de la integración de recursos-e}

Cada sistema de organización de recursos de información tiene ventajas e inconvenientes. Se pueden destacar como más importantes los siguientes:

1. Ventajas de la elaboración de bases de datos de recursos digitales:
- Inmediatez de localización de la información una vez que se ha elegido la herramienta de búsqueda.

- Son bases de datos especializadas en un campo concreto del conocimiento y están dirigidas a segmentos particulares de miembros de la comunidad de usuarios.

-Facilitan el acceso a numerosos recursos a partir de una materia o tema específico.

- Son seleccionados por profesionales y pasan a formar parte de la base de datos después de verificar que cumplen unos mínimos criterios de calidad: contenido, autoría, ergonomía, representación de la información, etc. (Codina, 2000).

\section{«Hay librerías electrónicas co- mo Amazon, Barnes \& Noble o Powell's books que funcionan, en cierto modo, como un opac extendido»}

2. En cuanto a los inconvenientes podríamos enumerarlos de la siguiente manera:

- Su proceso y tratamiento se realiza de manera independiente por lo que los métodos de búsqueda y recuperación de información cambian con respecto al opac y, además, no es fácil realizar el intercambio de registros bibliográficos entre otras bibliotecas y el sistema integrado de gestión bibliotecaria (sigb).

- Los recursos digitales no se relacionan con el resto de fuentes de información que posee la biblioteca al no estar incluidos ni reflejados en el catálogo.

-Este sistema contribuye a la dispersión y desorientación que sufre el usuario a la hora de la búsqueda y elección de las herramientas adecuadas para recuperar información.

-Restringe el ámbito de conocimiento o área de investigación del usuario pues suelen ser muy especializados.

3. Ventajas de la integración de recursos web en el opac:

- Ya se ha comentado que, hoy en día, las colecciones del fondo bibliográfico de cualquier biblioteca están formadas tanto por materiales en soporte tangible como por documentos digitales. Ambos tipos de recursos pasan por una serie de procesos de gestión: selección, adquisición, catalogación, indización, clasificación, etc. La inclusión de los documentos digitales en el opac hace que sean procesados de una manera semejante, uniforme y normalizada al resto de la colección, con lo que se puede considerar una ventaja de ca- 


\section{SISTIEMIA SAIBIINI}

\section{Soluciones integrales para la Automatización de Bibliotecas y Centros de Documentación}

Desarrollado integramente en castellano con más de 15 años de experiencia en España y

Latinoamérica, atiende las necesidades de todo tipo y tamaño de bibliotecas y centros de información

MÓDULOS:

- Adquisiciones

Trămite de pexfidos

Gesticon de gastos y proveedores

- Catalogación

Catalogacion de todos los materlales biloliográticos Integración de información bibliográfica

- Terminologia

Ficheros de auloridades

Tesauro multiingüe

Lenguaje de Comandos

- Consultas

Acceso público en linea (CAPEL)

Acceso a través de WWW

Protocolo 739.50

- Circulación

Gestión de préstamos

Fichero de usuarios

- Estadísticas

De proceso y circulación

Otros servicios:

Instalación y soporte técnico del sistema SABINI

Asesoria en Automatización de Bibliotecas y Centros de Documentación

Procesamiento de material bibliográfico

Instalación de catálogos en Internet de Bibliotecas
Cr Amar de Dica, $\mathrm{n}^{*} 1$ Tolf.: +34 91429055 $\mathrm{Fax}+34914292010$ 20014 MMRID F-mal: sabunfistabion

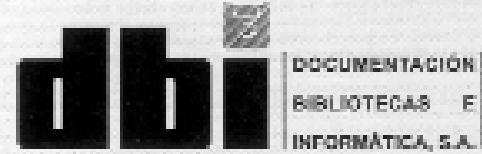

Av. Diez Cinseco Zse of. 602 Lims 18 Twhere: (511) 446-0815 en-mail: anbinipterra.compe 
ra a una posterior recuperación o intercambio de información y registros bibliográficos entre sistemas.

-Mayores relaciones entre los documentos individuales incluidos en el opac con el fondo total.

-Eliminación de la dispersión y multiplicidad de formas de acceso a un mismo recurso: desde el opac, desde la base de datos independiente del catálogo o desde guías temáticas de recursos digitales.

\section{Inconvenientes de esta unión:}

- Supone adaptar el sistema Dublin Core y sus 15 elementos básicos — ver pp. 450 - para describir recursos digitales de manera que permita la descripción para su plena integración en el opac junto a registros en formato marc.

- Conlleva una importante labor y un gran esfuerzo de mantenimiento del control de autoridades (autores personales, entidades, títulos uniformes y materias) para que las referencias e hiperenlaces estén bien definidos.

Hay que señalar que, frente a las ventajas que se derivan de la integración de recursos digitales en el opac, no es la opción más frecuente de momento. Hasta ahora las bibliotecas ya han integrado el acceso a revistas-e y bases de datos, pero continúan ofreciendo un acceso complementario desde páginas web temáticas.

\section{Proyectos de integración de recursos electrónicos en el opac}

Algunas de las actuaciones ya realizadas en este sentido en las bibliotecas serían (Estivill, A.; Abadal, E., 2000):

-Internet Resource Project de Oclc. Este proyecto experimental fue decisivo para el desarrollo del campo 856 del formato marc de localización y acceso a recursos electrónicos.

-Internet Cataloging Project, bajo la responsabilidad de Oclc, fue desarrollado en 1995 con el objetivo de aplicar las normas y reglas de catalogación al tratamiento de materiales y recursos digitales, para detectar así los problemas que surgieran. Se elaboró una base de datos de recursos electrónicos catalogados ( $I n$ terCAT) que fue operativa hasta 1999 y que después pasó a engrosar el catálogo colectivo WorldCat.

-NetFirst. Los responsables de alimentar con registros bibliográficos esta base de datos forman parte de la propia plantilla de Oclc y la descripción bibliográfica de cada documento se acompaña de un resumen, una indización según la Library of Congress subject headings (Lcsh), una clasificación según la $D D C$ (Dewey decimal classification) y un enlace a través de la url. Aunque se creó con un formato propio, desde 1999 se convirtió a marc.

-Corc. Cooperative online resource catalog es el último proyecto llevado a acabo por Oclc y en sus inicios fue un catálogo de recursos internet en el que se mezclaban registros en formato marc y otros con metadatos - tomando como base el Dublin Core y las pautas del RDF (resource description framework) para el intercambio de metadatos y el lenguaje de marcado xml- Este proyecto dio lugar a la creación del campo 856 "Electronic location and access» en el usmarc. Corc es un claro ejemplo de que el modelo de bases de datos de recursos digitales y las propias fuentes digitales integradas en el opac son complementarias y no puede afirmarse que una opción excluya a otra. Además ha demostrado que un registro en marc o en Dublin Core puede convertirse fácilmente en otro, lo cual significa que la compatibilidad entre sistemas está garantizada.

Si se consigue utilizar la definición de un conjunto de metadatos para la descripción de recursos internet, cada biblioteca se ahorrará la ardua tarea de crear e implementar proyectos locales. Además, se logrará compartir e intercambiar información bibliográfica del mismo modo que con el formato marc, aunque siempre habrá que revisar la indización y clasificación pues en estos elementos no existe tanta uniformidad.

\section{Elementos necesarios para el diseño del nuevo opac}

Para conseguirlo serán necesarios elementos tecnológicos superiores a los que tenemos en la actualidad. La aplicación informática que contenga el módulo del opac sería un sistema basado en arquitectura cliente/servidor que corra, por ejemplo, bajo plataforma Unix, que utilice Oracle como gestor de bases de datos y $B R S /$ Search para buscar texto completo dentro del propio catálogo; será necesario el empleo de metadatos para la catalogación; la aplicación de algún tipo de «inteligencia artificial» en la realización de búsquedas de información más complejas y potentes; el uso del lenguaje de edición xml para el intercambio de datos e información; la implementación de sistemas de codificación como Unicode (que proporciona un número único para cada carácter sin importar plataforma, programa o idioma que, además, es un requisito para los estándares modernos como xml, Java, etc., y es la manera oficial de aplicar la norma ISO/IEC 10646).

Resultará útil también añadir los servicios propios del doi. El empleo de utilidades como CrossRef permite enlazar las citas con los contenidos o textos completos de cada documento mediante una base de datos central en la que los editores incluyen metadatos como 
los asignados a sus artículos publicados. El uso de aplicaciones como Hyperion, que ofrece un acceso ordenado y seguro a las fuentes informativos disponibles en soporte digital, facilitando además la creación de catálogos de recursos documentales disponibles en formato digital: imágenes digitalizadas, archivos pdf, vídeos, sonido, documentos con marcas html, sgml, etc., así como la gestión de los metadatos asociados a cada uno de ellos utilizando el conjunto Dublin Core para la descripción de recursos digitales. Habrá que tener previsto el almacenamiento de información en servidores de gran capacidad, la implementación de robots que vayan comprobando enlaces y generen listados de los que fallan, etc. En definitiva, los sigb deben entrar de lleno en la era electrónica, permitiendo catalogar y dar acceso directo a información electrónica, libros-e, periódicos-e, vídeos, sonidos en formato wav, mp3, etc. (Gethin, 2001).

Las posibilidades de contacto y comunicación del usuario con la biblioteca que ofrecen los actuales catálogos (consultar la situación del usuario; renovación de préstamos; visualización de nuevas adquisiciones, envío de desideratas; envío de mensajes; solicitud de información bibliográfica) se mantendrían, pero añadiendo las nuevas posibilidades que ofrece la telefonía como por ejemplo: reservas de libros desde el wap del móvil, envío de mensajes sms (short message service) al usuario, etc.

Esta nueva herramienta mantendría los ti-

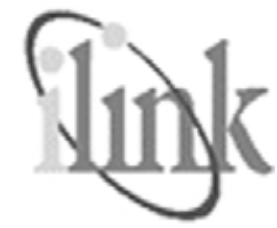
pos de búsquedas que actualmente ofrece cualquier opac: búsqueda sencilla o básica, compleja o avanzada, por signatura topográfica o por el sistema de clasificación de los materiales. Pero, además, debiera incorporar una consulta por asignatura (muy útil para las bibliotecas universitarias) y desde la que se accediera a los distintos recursos informativos, en papel o en formato digital, necesarios para seguir cualquier asignatura de cada una de las titulaciones impartidas en una universidad. Un buen ejemplo de esta aplicación es la opción que presenta el catálogo de la biblioteca de la Universitat Oberta de Catalunya.

http://www.uoc.es/

Será necesario analizar y valorar la potencialidad del lenguaje de recuperación: los operadores que se pueden utilizar, la posibilidad de repetición de estrategias ya realizadas, los enlaces hipertexto, la búsqueda relacional o en varios catálogos a la vez, etc. Habrá que tener en cuenta al introducir los términos de consulta algunas reglas como: que el sistema no distinga en el uso de mayúsculas y minúsculas; que puedan ser tecleados con o sin acentos; el uso de apóstrofes; las palabras vacías que sean ignoradas por el sistema; que el sistema opac tenga en cuenta los espacios en blanco, etc. En cuanto a los operadores, cualquier sistema debe tener básicamente los siguientes tipos: booleanos (and, or, not xor —or exclusivo-), posicionales (same, with, near, adj) y truncamientos.

\section{ILink: un posible opac-portal}

Un ejemplo de este nuevo opac-portal con servicios para bibliotecas universitarias y especializadas puede ser iLink ${ }^{1}$. Ha sido desarrollado por Sirsi Corporation $\mathrm{y}$, de igual manera que iBistro está diseñado para atender las necesidades de los usuarios de las bibliotecas públicas, iLink pretende ofrecer una vía de acceso única para la búsqueda, presentación y recuperación de información en bibliotecas académicas y científicas.

\section{http://ibistro.sirsi.com/uhtbin/cgisirsi/A6wtNzF1fI/22 1370007/60/100/X}

ILink permite la consulta en el propio catálogo del centro, en los de otras bibliotecas mediante Z39.50, en bases de datos en línea y, además, en cualquier web de internet. La novedad es que incluso hace posible realizar la consulta simultánea en todas estas fuentes de información desde una misma ventana de búsqueda. Los amplios resultados que se obtengan después de llevarlas a cabo se podrán organizar y estructurar en categorías más concretas y específicas, agrupando los resultados por submaterias.

Su servicio Mylink funciona como un mecanismo de difusión selectiva de la información y permite crear portales personalizados de conocimiento de acuerdo con los intereses de cada usuario: listas especializadas de materias, autores, novedades, actividades de la biblioteca, etc. Además el opac-portal ofrecerá valiosa información adicional como críticas de libros, sumarios, primeros capítulos de obras, índices, biografías de autores, etc.

La opción Link's digital media archive funciona como un contenedor o depósito de archivos informáti$\cos$ y digitales. Permite preservar y almacenar una gran variedad de información incluyendo imágenes y/o textos digitalizados, documentos en pdf, ascii, $\mathrm{html}$ o sgml; ficheros de audio (wav, aiff, au, mp3) o de vídeo (avi, mpeg, mov).

\section{Nuevas tendencias y algunas formas de expandir los opacs}

Existen múltiples procedimientos de ampliar el ámbito de actuación de los opacs que superan los límites de los tradicionales catálogos bibliográficos. Algunas de estas posibilidades son las siguientes: 


\section{Acceso a recursos de información/documentos.}

a. Proporcionar acceso a contribuciones de obras colectivas: actas, comunicaciones y ponencias de congresos, recopilaciones de trabajos académicos, libros homenaje, antologías, etc. Hoy en día se podría ofrecer esta opción mediante el uso de la catalogación analítica de cada una de las partes que componen el documento, añadiendo un enlace desde una url que aparezca en el registro bibliográfico que muestra el opac hasta el texto digitalizado de la parte integrante del documento general. Esta idea está relacionada directamente con el nuevo concepto de "granularidad" (el nivel de especificidad precisado para identificar y describir las partes de un documento digital), que se perfila como una de las posibles opciones para este servicio.

b. Ofrecer enlaces a monografías que se encuentran en formato electrónico. Por el momento, una solución puede ser la de vincular el opac al catálogo de alguna biblioteca virtual de manera que se pudieran obtener referencias bibliográficas que ofreciesen el acceso al texto completo de los documentos digitalizados.

c. Acceso a recursos electrónicos de internet: selección y compilación de sitios web sobre distintas materias. Los registros descriptivos de los documentos electrónicos deberían contener los siguientes 7 metadatos básicos: título, tipo de recurso, responsabilidad, lugar, editor, número normalizado y url. Aunque siempre se podría ampliar el conjunto de elementos hasta llegar a los 15 que componen el Dublin Core y que son los siguientes: título, autor o creador, materias y palabras clave, descripción, editor, otras contribuciones, fecha, tipo de recurso, formato, identificación del recurso, fuente, idioma, relaciones, alcance y gestión de derechos.

Se trataría de recursos digitales considerados de interés y lo novedoso sería poder catalogarlos y ofrecerlos directamente desde el opac de la biblioteca y no independientemente como aparecen en la mayoría de los webs de bibliotecas universitarias.

d. Proporcionar acceso al texto completo de la normativa y legislación de la propia universidad a la que dé servicio la biblioteca. Se recogería la normativa en una sede web y se cargaría en el opac para tener un acceso directo desde éste.

e. Ofrecer la consulta y el préstamo de los libros-e. Estos documentos se almacenan físicamente en los servidores de que disponga la biblioteca y deben ser desprotegidos por el mecanismo de lectura del usuario cuando sean prestados. Se descargan en un pc y el dispositivo de visualización flexible se puede enrollar para transportarlo. Las bibliotecas pueden hacer suscripciones que permitan a un determinado número de usuarios obtener ejemplares prestados del libro-e en períodos de tiempo simultáneos. Por ejemplo, NetLibrary es un servicio que ofrece libros-e. Otros productores de libros electrónicos son: Books.com (Barnes \& Noble) y Cytale.

http://www.netlibrary.com/

http://ebooks.bn.com

http://www.cytale.com

f. Acceso a los índices de recursos y motores de búsqueda de internet desde la misma página del opac como complemento a las herramientas propias de búsqueda de información que ofrezca la biblioteca.

\section{Utilidades de la biblioteca.}

a. Acceso en línea al sistema de clasificación que utilice para organizar sus fondos, de manera que el usuario pueda saber qué números de clasificación corresponden a la materia de los documentos en los que pueda estar interesado.

b. Acceso en línea a las tablas de materias que se empleen en la biblioteca para indizar los documentos. El usuario conocerá cómo se construyen los encabezamientos de materia asignados y, hasta que no esté más estandarizado el uso del lenguaje natural en la recuperación de información a través de opac, le puede resultar muy útil. Sería recomendable la superación de estos esquemas tan rígidos de indización en favor del empleo de los índices de palabras clave y tesauros. Esto mejorará y facilitará tanto la cooperación internacional como el intercambio de información entre instituciones de diferentes países. Además, los tesauros no limitarían tanto las relaciones y vínculos que se establecen entre los términos del lenguaje de recuperación.

\section{Otros servicios.}

a. Selección de los libros más leídos cada mes: daría una idea del tipo de obras (manuales, monografías científicas, obras de creación, de consulta generales) que se están consultando y circulando con más frecuencia. Así podríamos conocer mejor los perfiles de interés de nuestros usuarios.

b. Tema del mes: ofrecer una relación de lecturas recomendadas y otro tipo de recursos digitales relacionados con un tema concreto de actualidad que se determine mensualmente.

c. Posibilidad de solicitar documentos por préstamo interbibliotecario a través del opac: los formularios de petición de documentos estarían accesibles desde una página web colgada del catálogo.

d. Elaboración de foros de discusión y charla sobre temas literarios, científicos, etc., teniendo presente el acceso a ellos por medio del opac. 
e. Dar la posibilidad al usuario de crearse una página web personalizada. Se le asignaría un acceso particular por medio de un número de identificación y una contraseña.

\section{Prestaciones/utilidades presentes en los opacs actuales}

-Proporcionar acceso a artículos de revista. Actualmente la solución empleada es usar el campo de acceso electrónico, etiqueta 856 del formato marc, para incluir el url de la publicación. Sin embargo, la tendencia es utilizar las aplicaciones de la granularidad o buscando directamente por doi (número que identifica todo tipo de recurso papel o digital; se aplica a cualquier grado de especificidad de contenido e, incluso, permite buscar el recurso directamente desde el navegador). Lo más útil sería poder llegar a la referencia bibliográfica y a continuación enlazar desde el título del artículo al texto digitalizado, vincular desde el autor al resto de sus obras, desde el nombre de la publicación hasta la ficha bibliográfica completa y desde ésta al texto íntegro de la publicación.

\section{«Las bibliotecas ya han inte- grado el acceso a revistas-e y bases de datos, pero continúan ofreciendo un acceso comple- mentario desde páginas web temáticas»}

- Suministrar acceso a obras de referencia: catálogos, diccionarios, enciclopedias, etc. Las obras de consulta general que existen en formato electrónico se podrían catalogar e indizar incluyéndose en el opac de manera que apareciese en el registro bibliográfico el enlace a la sede web en la que se pudieran consultar. Esta opción contrasta con la solución que hoy en día ofrecen la mayoría de centros tras el enunciado "referencia electrónica" que, aunque no es un error presentarla como tal, resultaría mucho más práctica incluida además en el propio catálogo de la biblioteca.

- Proporcionar acceso a bases de datos. Se deberían catalogar estas fuentes en formato marc añadiendo un enlace que apareciese en un campo del registro bibliográfico para permitir la conexión desde el mismo opac y no separadamente. Actualmente muchas bibliotecas lo hacen desde su página web, pero de forma independiente al catálogo. Se presentan por orden alfabético de títulos, por materias, por la vía de adquisición: suscritas, de libre distribución en internet, etc., pero no siempre se ofertan incorporadas también en el catálogo de la biblioteca como otro de los muchos recursos que ésta ofrece para resolver y satisfacer las necesidades de información.
- Permitir la consulta de catálogos y bases de datos de otras bibliotecas y también de las que, por ejemplo, se hubiera formado un consorcio. El acceso a catálogos se puede hacer por medio del protocolo Z39.50 (norma ISO 23950), un estándar internacional para la comunicación entre sistemas informáticos. Permite realizar búsquedas de información en distintas bases de datos empleando un único lenguaje de recuperación (e independiente del propio de cada aplicación), presentando una interfaz semejante y presentando los resultados uniformemente, además de suponer un gran ahorro de tiempo en las consultas. Una de sus ventajas radica en que no es necesario que el usuario conozca perfectamente la sintaxis de interrogación de cada base de datos. Además se trata de un protocolo abierto, basado en arquitectura cliente/servidor, que permite a un ordenador cliente (origin) buscar en bases de datos de un ordenador servidor (target) pues conecta aplicaciones de distintos sistemas.

Como inconvenientes cabe señalar que, al tratarse de una norma muy amplia, cada servidor y cada cliente ha implementado distintas opciones por lo que a veces surgen incompatibilidades. Además, muchos de los nuevos servicios y aplicaciones todavía no están siendo utilizados. Existen problemas con los niveles de servicio soportados dentro y entre una implementación particular del cliente y el servidor $Z$, así como con las capacidades y la implementación del host del sigb. Aunque Z39.50 permite búsquedas en bases de datos comerciales como Dialog, Lexis-Nexis, etc., puede plantear dificultades a la hora de aplicarlo como pasarela de consultas en otras aplicaciones más complejas. También hay que señalar su incompatibilidad con funciones de búsquedas en tesauros.

Una posible opción es crear una web con una imagen del mapa de España por comunidades autónomas desde el que se enlazara a catálogos de bibliotecas universitarias, virtuales, consorcios, Biblioteca Nacional, etc. También se debería dar conexión a los opacs de otros centros extranjeros.

- Acceso a determinadas bases de datos creadas en la biblioteca con materiales particulares de la institución. Por ejemplo: a la base de datos de tesis doctorales; acceso a los materiales de aprendizaje de idiomas que existen en el laboratorio o en la universidad, etc.

-Ofrecer el acceso a los enunciados de exámenes: digitalizar a texto completo los de las distintas convocatorias de cada asignatura y ponerlos en la Red. También incluir la resolución de cada ejercicio para que los alumnos puedan bajar directamente estos materiales del opac y consultarlos. 
-Enlazar con los apuntes y prácticas propuestas por los profesores de cada asignatura de las titulaciones impartidas en la universidad.

- Pactar acuerdos con el servicio de publicaciones de la universidad para poder dar acceso al texto completo de los materiales que publica. La UPC ya ofrece el acceso a este tipo de materiales de esta forma.

\section{http://bibliotecnica.upc.es/EdUPC/inici.asp}

- Añadir en la descripción bibliográfica de cada documento en papel un enlace al índice escaneado del documento y al resumen del libro para ampliar la información que ofrece cada registro bibliográfico.

\section{Elementos de comunicación directa entre la biblioteca y el usuario.}

a. Crear perfiles temáticos por usuario para recibir información periódica acerca de las novedades que la biblioteca ha recibido relacionadas con los intereses que cada uno haya establecido previamente. Así el opac ofrecería un sistema de alerta sobre novedades o sus preferencias informativas.

b. Dirigir consultas al personal de la biblioteca y solicitar todo tipo de información desde cualquier lugar e independientemente de cuándo lo haga. El opac funcionaría como un verdadero mostrador y punto de información y atención al público.

c. Enviar notificaciones a los usuarios desde la biblioteca vía correo-e o a través de mensajes a teléfonos móviles.

\section{Utilidades de la biblioteca.}

a. Tablón de anuncios de la biblioteca. Para dar a conocer las novedades y los últimos comunicados que se realicen. Muchos centros ya disponen de este tipo de tablón informativo. Un ejemplo es la biblioteca de la $U C 3 M$.

b. Exponer la normativa y/o reglamentos de la biblioteca en el opac-portal. Algunas ya la tienen accesible desde el web, como por ejemplo la Universitat Pompeu Fabra, que lo hace bajo la denominación de Carta de drets i deures dels usuaris.

http://www.upf.es/bib/cont $2 . h t m ? b=$ dalt $1 . h t m \& c=e s q$ 1.htm\&d=/bib/coneixer/normes/carta.htm

c. Elaboración de una web con una relación de las preguntas más frecuentes que los usuarios dirigen a los bibliotecarios. Este tipo de iniciativas se presentan ya en muchas webs de bibliotecas españolas como por ejemplo, la de la Escola Universitaria de Ciències Empresarials Dr. Manyà (adscrita a la Univ. Rovira $i$ Virgili) o la de la Universitat de Barcelona.

http://www.fudm.es/biblioteca/bca_pmf.html http://www.bib.ub.es/bub/faqs.htm

d. Publicar en la web del opac-portal las diferentes estadísticas de la biblioteca y los datos de su evaluación.

e. Ofrecer una visita virtual. Es lo que empiezan a ofrecer algunas bibliotecas en sus webs, un ejemplo lo constituye la $U C 3 M$.

f. Informar de la oferta de los cursos de formación de usuarios que el personal imparte.

g. Hacer públicos los horarios de apertura de la biblioteca y de los medios de transporte para llegar hasta la misma: líneas de metro o autobús, trenes, carreteras, etc. Por ejemplo, la biblioteca Etse-Etseq de la Universitat Rovira $i$ Virgili muestra un plano indicando cómo acceder a ella. Sería útil añadir también la información de acceso para las personas discapacitadas.

http://www.etse.urv.es/bibweb/

h. Dar a conocer a través de la web los servicios y facilidades que ofrece la biblioteca sobre ordenadores, conexiones a internet, servicios de impresión y fotocopiadora, de lectura y reproducción de microfilms, etc.

\section{A modo de conclusión: prioridades a la hora de integrar servicios}

La integración tanto de recursos como de servicios de información en el opac, bien organizada y estructurada, ayudará en la orientación al usuario a localizar y acceder a la información de manera ágil, fácil y provechosa. Si conseguimos organizar los productos que la biblioteca ofrece desde una misma sede web y enlazarlos con el opac, los usuarios cada vez serán más autónomos en la búsqueda de la información y además lo harán con mucha más destreza y satisfacción.

La mejora del acceso a los recursos de información es la principal tarea que se debe plantear el personal de la biblioteca. Se ha de ofrecer un acceso más completo a las obras colectivas, los libros-e, integración de recursos digitales, descarga de índices y resúmenes de los documentos desde el opac, digitalización de obras antiguas para su preservación así como difundir los materiales, etc. La comunicación entre la biblioteca y el usuario debe seguir creciendo. Es cierto que se ha mejorado considerablemente en este aspecto, pero todavía queda mucho por hacer: elaboración de perfiles temáticos sobre novedades, envío de notificaciones a los usuarios, dirigir consultas al bibliotecario, etc. La biblioteca debe facilitar al usuario toda la información acerca de los propios instrumentos que se utilizan para organizar, clasificar y acceder a la información, informar de las novedades, etc. Por último, necesita crecer en la oferta de servicios y no estancarse con los tradicionales, sino que necesita innovar con nuevas ide- 
as: selección de libros más leídos, relación de lecturas recomendadas sobre temas de actualidad, foros de discusión, personalización de webs particulares a partir del opac-portal, etc.

\section{Nota}

1. Ignoro el funcionamiento real de iLink pues únicamente he utilizado la aplicación de demostración que ofrece en su web la empresa Sirsi. Aun así, en mi opinión es un tipo de aplicación cuyo desarrollo debemos seguir atentamente.

\section{Bibliografía}

Arnedo, Txema. De portales a plazas; presente y futuro de los portales en internet. Consultado en: 23-03-02.

http://www.aui.es/biblio/libros/mi99/3portales.htm

Barnes S.; Mccue J. "Linking library records to bibliographic databases: an analysis of common data elements in Biosis, Agricola and the opac". En: Cataloging \& classification quartely, 1991, v. 13, n. 3-4, pp. 157187.

Boss, Richard W. "Online catalog functionality in the 90s: vendor responses to a model RFP (request for proposal)". En: Library technology reports, 1993 , v. 29 , n. 5, pp. 587-745.

Caswell, J. V., et al. "Importance and use of holding links between citation databases and online catalogs". En: Journal of academic librarianship, 1995, v. 21, n. 2, pp. 92-96.

Codina, Lluís. "Evaluación de recursos digitales en línea: conceptos, indicadores y métodos". En: Revista española de documentación científica, 2000, v. 23, n. 1, pp. 9-45.

Corc: cooperative online resource catalog. Oclc. Consultado en: 11-0402.

http://www.oclc.org/oclc/corc/index.htm

CrossRef. Consultado en: 17-04-02.

http://www.crossref.org/

Crawford, Walt. The online catalog book: essays and examples. New York: G. K. Hall, 1992.

Desire Project: development of an European service for information on research and education. Desire Consortium, c1998-2000. Consultado en: 16-04-02.

http://www.desire.org

DOI: digital object identifier. Consultado en: 17-04-02.

http://www.doi.org/

Dublin Core metadata initiative. DCI, c2000. Consultado en: 08-04-02. http://www.purl.org/DC/

Elib: the electronic libraries programme. Elib, última revisión: 6 de julio de 2000. Consultado en: 15-02-02.

http://www.ukoln.ac.uk/services/elib/

Estivill, Assumpció; Abadal, Ernest. "Acceso a los recursos web gratuitos desde las bibliotecas". En: El profesional de la información, 2000, noviembre, v. 9 , n. 11, pp. 4-20

Frías, José Antonio. "Expandiendo el opac: problemas y perspectivas". En: Jornadas bibliotecarias de Castilla La Mancha, 1998, pp. 281-284.

García Gómez, Juan Carlos; Saorín Pérez, Tomás. "Los portales de internet”. En: Curso de promoción educativa, 2001, mayo, Murcia.

García Testal, Cristina. "Corc: hacia la biblioteca digital”. En: El profesional de la información, 2000, abril, v. 9, n. 4, pp. 17-20.

García Testal, Cristina. "Digital object identifier". En: El profesional de la información, 2001, agosto, v. 10, n. 7-8, pp. 26-31.

Gethin, Peter. "Automatización de bibliotecas". En: El profesional de la información, 2001, noviembre, v. 10, n. 11, p. 26-31.

Gethin, Peter. "Bibliotecas y tecnología: entrevista con Peter Gethin". En: El profesional de la información, 2000, junio, v. 9, n. 6, pp. 26-34.
Gorman, Michael. "The future of cataloguing and cataloguers". En: Ifla general conference, 1997, Booklet 4, Division of Bibliographic control, pp. 37-38.

Hildreth, Charles R. Online catalogue: developments and directions. London: The Library Association, 1989.

Hildreth, Charles R. Online public access catalogs: the user interface. Dublin, Ohio: Oclc, 1981.

Hyperion. Consultado en: 28-01-02.

http://www.sirsi.com/Sirsiproducts/hyperion.html

IBistro. Consultado en: 12-12-01.

http://library.ncsc.dni.us/uhtbin/cgisirsi.exe/x/0/49

ILink. Consultado en: 12-04-02.

http://ibistro.sirsi.com/uhtbin/cgisirsi/A6wtNzF1fI/221370007/60/100/X

IMesh: International collaboration on internet subject gateways. Uk office for library and information networking, University of Bath, 3 de septiembre de 1999, última actualización: 20 de junio de 2000. Consultado en: 10-03-02.

http://www.imesh.org/

InterCAT project. OCLC. Consultado en: 09-04-02.

http://www.oclc.org/oclc/research/projects/intercat.htm

Jiménez, Miguel. "Las nuevas tecnologías de la información en las bibliotecas universitarias. La ventanilla única”. En: Curso selectivo de promoción interna para el acceso a las escalas técnica, archiveros, bibliotecarios y documentalistas, 2001, Alicante.

Jiménez, Miguel. "Una norma para la cooperación Z39.50”. En: Actas de las I Jornadas bibliotecarias de Castilla La Mancha, 1998, pp. 265-277.

Knudson, Frances L., et al. "Creating electronic journal web pages from opac records". En: Issues in science and technology librarianship, 1997, n. 15. Consultado en: 04-04-02.

http://www.library.ncsb.edu/istl/97-summer/article2.html

Machovec, George S.; Brunning, D. R. "The online catalog as a shared resource: the impact of local databases and networking". En: Advances in library resources sharing, 1991, 2, pp. 87-99.

Marcos, Mari Carmen. "Diversos modos de acceso a catálogos en línea a través de internet". En: El profesional de la información, 1999, enerofebrero, v. 8, n. 1-2, pp. 7-14.

Morrison, David. Building successful portals. Consultado en: 23-03-02. http://www.e-

promag.com/eparchive/index.cfm?fuseaction $=$ viewarticle $\&$ ContentID $=11$ $0 \&$ publicationid $=1 \&$ channel $=2$

Ortiz-Repiso Jiménez, Virginia. "Nuevas perspectivas para la catalogación: metadatos versus marc". En: Revista española de documentación científica, 1999, v. 22, n. 2, pp. 198-219.

Peis, Eduardo; Fernández-Molina, Juan Carlos. "Los opacs supermercados de información bibliográfica". En: Jornadas bibliotecarias de Andalucía, 1996, pp. 468-475.

Porter, G. Margaret; Bayard, Laura. "Including web sites in the online catalog: implications for cataloging, collection development, and access". En: The journal of academic librarianship, 1999, septiembre, v. 25, n. 5, pp. 390-394.

Taylor, Arlene G.; Clemson, Patrice. "Access to networked documents: catalogs? search engines? both?. En: Oclc internet cataloging colloquium, 1996. Consultado en: 01-02-02.

http://www.oclc.org/oclc/man/colloq/toc.htm

Unicode. Consultado en: 19-11-01.

http://www.unicode.org/unicode/standard/translations/spanish.html

Z39.50. Consultado en: 17-04-02.

http://www.ifigenia.es/zeta/z391.htm

$M^{a}$ Victoria Játiva Miralles, Universidad de Alicante. victoria.jativa@ua.es 